\title{
Intraoperative cortical mapping of visuospatial functions in parietal low-grade tumors: changing perspectives of neurophysiological mapping
}

\author{
Andrea Talacchi, M.D., ${ }^{1}$ Giovanna Maddalena Squintani, M.D., ${ }^{2}$ \\ Barbara Emanuele, Ph.D., ${ }^{3}$ Vincenzo Tramontano, Ph.D., ${ }^{1}$ Barbara Santini, Ph.D., 1 \\ and Silvia Savazzi, Ph.D. ${ }^{3}$ \\ Sections of ${ }^{I}$ Neurosurgery and ${ }^{3}$ Physiology and Psychology, Department of Neurological, \\ Neuropsychological, Morphological and Movement Sciences, University of Verona; and ${ }^{2}$ Unit of Neurology, \\ Department of Neurosciences, University Hospital, Verona, Italy
}

\begin{abstract}
Object. The aim of this study was to explore the feasibility of intraoperative visuospatial mapping with the same criteria currently used to define essential language areas.

Methods. The authors compared surgical procedures in 2 patients with similar tumors (Grade II oligodendroglioma in the right parietal lobe) undergoing awake, image-assisted surgery for lesion removal with intraoperative neurophysiological monitoring. The line bisection task was used in both patients but with different criteria.

Results. In the first case, the authors respected any area, even within the tumor, where significant interference was found (a stimulation-induced error in 2 of 3 applications defined an essential area). In the second case, they removed 1 essential area located in the tumor and recorded an uneventful clinical response soon thereafter. They continued to monitor the patient without stimulation and stopped the resection when the patient was close to the criteria valid for defining spatial neglect. The signs of spatial neglect were present for 3 days postoperatively and then cleared spontaneously. Subtotal tumor removal was achieved in both cases.

Conclusions. Evidence in the present study reveals that areas for visuospatial functions cannot be assessed with the same criteria used for language functions, since essential areas located in the tumor can be safely removed. (http://thejns.org/doi/abs/10.3171/2012.12.FOCUS12358)
\end{abstract}

\section{KEY WORDS • glioma - visuospatial function • neuropsychological assessment • patient outcome assessment}

$\mathrm{N}$ EUROPHYSIOLOGICAL mapping provides considerable advantages in brain tumor surgery: it extends the surgical indications and improves the quality of tumor resection by highlighting eloquent areas of the brain, which are preserved while adjacent tumor is safely removed. ${ }^{3}$ To date, although there is consistent experience with motor and language mapping, attempts to map other functions are still limited by several methodological issues: lack of appropriate tasks, the different patterns and complexity of neurophysiological circuits, uncertainty about the correspondence between focal injury and permanent clinical impairment as well as false-negative results, and the reliability of the same criteria for both motor and language mapping. ${ }^{6,11}$

In large, low-grade right parietal lobe tumors, the risk of causing spatial neglect is difficult to predict and argues against surgery. ${ }^{9,10}$ Here we describe our experience with 2 different mapping strategies. In the first case we applied the same criteria used in language mapping and preserved an essential area within the tumor. In the second case we removed one essential site within the tumor.

\section{Methods}

Two patients who underwent surgery for a right parietal lobe glioma took part in this study. The patients gave written informed consent to undergo the procedure, which was performed in accordance with the ethical standards laid down in the 1964 Declaration of Helsinki. Both patients were right-handed, as indicated by the Edinburgh Handedness Inventory. They both underwent a preliminary multidisciplinary interview as well as a battery of psychological and neuropsychological tests (Table 1) before and after surgery. ${ }^{12,14,15}$ In addition to general neuropsychological testing, assessments of their spatial abilities and spatial neglect in particular were performed. ${ }^{4}$ We followed the standard rules to assess spatial neglect: for all the paper-and-pencil tests, the sheet of paper was preoperatively centered to the patient's sagittal head plane, and no time limit was given for task execution (except for the Diller Letter Cancellation Test, when the time limit was 2 minutes). ${ }^{1}$

To familiarize patients with the task they would per- 
form during the operation, prior to surgery they were asked to bisect a series of black horizontal lines $20 \mathrm{~cm}$ long and 2 $\mathrm{mm}$ thick. Each line was centered on a horizontal standard (A4) sheet of paper, and the leftmost border of the sheet of paper was aligned to the patient's sagittal head plane. The patients were instructed to mark the center of each line using a pencil. This task was done to obtain a more conservative measure of neglect errors; that is, the patients' pathological performance should be underestimated given that displacing the line to the right results in a decreased rightward error. Patients performed the same task during surgery, but electrocortical stimulation was applied during the intraoperative task. Prior to testing, the neurosurgeon labeled with numbers several cortical sites at the tumor location and different areas around the tumor. Cortical mapping was initiated at a low stimulus intensity $(2 \mathrm{~mA})$ and was continued at increments of $2 \mathrm{~mA}$. The test was defined as positive for essential areas when errors were caused by at least 2 of 3 stimulations applied at the maximum intensity below the afterdischarge threshold observed on electrocorticography. Patients and examiners were unaware of the electrocortical stimulation efficacy. After each bisection, an independent examiner reported the number of the stimulated site to keep track of the total number of stimulations for each site, and another examiner assessed the accuracy of the bisection mark. This latter step was done by overlapping the test sheet with a transparency paper indicating the $5 \%(10 \mathrm{~mm})$ and $10 \%(20 \mathrm{~mm})$ deviations. When a deviation to the right of the geometric center was greater than $5 \%$, a rightward bisection error was recorded for that site. When a bisection error occurred, the patients were asked to bisect other lines without further stimulation until their performance reverted to normal. When the bisection error returned to normal, a new site was stimulated.

\section{Illustrative Cases}

\section{Case 1}

History and Examination. A 47-year-old man in otherwise good health experienced an epileptic attack characterized by sudden paresthesia of the left upper limb, associated with general malaise, and followed by headache. The episode cleared up within 2 hours, but the patient reported light paresthesia for the next 3 days. On MRI, T1-weighted sequences showed a parietal hypointense area, which was better visualized on T2-weighted sequences as a hyperintense corticosubcortical lesion. The core lesion measured $4 \mathrm{~cm}$ with very irregular margins and gradual centrifugal modification of the signal. Spectroscopy displayed discrete intralesional areas with an inverse choline/ $N$-acetylaspartate ratio, while a perfusion imaging modality depicted a slight increase in intralesional regional cerebral blood flow.

Because of findings suggestive of high-grade glioma, the patient underwent intraoperative neurophysiological monitoring and image-assisted surgery. Preoperative assessment showed that intelligence, attention, verbal and visuospatial memory, and instrumental functions (language, praxis, and gnosis) were intact, but executive functions (FAS Test) were impaired (Table 1).
Operation. Throughout the operation, the patient was kept awake. Neuronavigation was used to visualize the edges of cortical tumor and identify anatomical landmarks. The exposed cortical area included the superior temporal, angular, supramarginal, infraparietal, and postrolandic gyri. Under continuous electrocortical recording (using a 6-electrode strip), phase reversal was initially assessed to localize the prerolandic area, and the residual area was mapped using the line bisection task to recognize putative areas of spatial awareness. Two (Labels 4 and 5 in Fig. 1B) of 11 areas were essential at a stimulus of $8 \mathrm{~mA}$, overlapped the tumor area, and were preserved. At the anterior tumor edge, motor evoked potentials from the face were found and represented another limit of resection.

Postoperative Course. Postoperative MRI confirmed removal of the central part of the tumor, accounting for about $60 \%$ of the lesion area. Postoperative assessment revealed unchanged word fluency impairment (FAS Test) and a decline on the visuospatial memory test (Spatial Supraspan Learning; Table 1). At 3 months after surgery, all tests results were within normal limits. Histological examination revealed a WHO Grade II oligodendroglioma with deletions on both $1 \mathrm{p}$ and $19 \mathrm{q}$.

Case 2

History and Examination. A 44-year-old woman experienced epileptic seizures initiating as an electric shock running from the left foot to the face. Because the patient was pregnant, she waited until the 6th month of pregnancy before starting therapy. She had experienced 3 epileptic attacks prior to therapy, one of which was characterized by clonic tremor of the left hand. A T2-weighted MR image showed a postrolandic tumor as a hyperintense area with irregular margins. Spectroscopy displayed an inverse choline $/ N$-acetylaspartate ratio in the tumor center. Preoperative assessment revealed that intelligence, executive functions, attention, verbal memory, and instrumental functions (language, praxis, and gnosis) were intact, whereas visuospatial memory was impaired (Spatial Supraspan Learning and Rey-Osterrieth Complex Figure Test, recall condition).

Operation. After delivery of her child, the patient underwent surgical removal of the tumor. The procedures were similar to those in Case 1: awake surgery, neuronavigation, intraoperative electrocorticography and cortical mapping with stimulation during the line bisection task, phase reversal, and motor evoked potentials monitoring. We identified tumor in the supramarginal and angular gyri. Three sites (Labels 0,3 , and 5 in Fig. 2B), two of which were inside the tumor area (Labels 0 and 3), were essential, since errors were induced 2 and 3 times, respectively, upon 3 stimulations; 4 sites were negative (6 mA). In this patient, we took a new approach: we started the resection at sites where the mapping was negative, planning to later include essential areas; after resecting each site, we repeated the behavioral task without electrocortical stimulation. We stopped the resection when the spontaneous error was $7 \mathrm{~mm}$ (that is, after resection of the area labeled as 0 in Fig. 2B), near the cutoff for neglect (10 


\section{Intraoperative visuospatial mapping for glioma}

TABLE 1: Psychological and neuropsychological tests (corrected scores)*

\begin{tabular}{|c|c|c|c|c|c|}
\hline \multirow[b]{2}{*}{ Test } & \multirow[b]{2}{*}{ Cutoff Score† } & \multicolumn{2}{|c|}{ Before Surgery } & \multicolumn{2}{|c|}{ After Surgery } \\
\hline & & Case 1 & Case 2 & Case 1 & Case 2 \\
\hline Edinburgh Handedness Inventory & $<40 / 100$ & $40 / 100$ & $100 / 100$ & NE & NE \\
\hline Beck Depression Inventory & $\geq 14$ & 5 & 5 & 5 & 4 \\
\hline STAI-Y, state anxiety & NV $43 \pm 12$ & 51 & 50 & 44 & 49 \\
\hline STAI-Y, trait anxiety & $43 \pm 9$ & 49 & 47 & 48 & 47 \\
\hline Pain Anxiety Symptoms Scale & $>56.6$ & 34 & 21 & NE & NE \\
\hline Raven Colored Progressive Matrix 47 & $\leq 18.96$ & 30.3 & 28.3 & 27.3 & 29.3 \\
\hline verbal fluency (FAS Test) & $\leq 17.35$ & $14 \S$ & NE & $15 \S$ & NE \\
\hline Trail Making Test-A & $\geq 94$ & 49 & 40 & 66 & 45 \\
\hline Trail Making Test-B & $\geq 283$ & 151 & 175 & 180 & 172 \\
\hline Forward Digit Span Task & $\leq 3.75$ & 4 & 5 & 4 & 5 \\
\hline Backward Digit Span Task & $\leq 3.75$ & 4 & 5 & 4 & 4 \\
\hline 15 Rey Auditory Verbal Learning, immediate recall & $\leq 28.53$ & 38 & NE & 35 & NE \\
\hline 15 Rey Auditory Verbal Learning, delayed recall & $\leq 4.69$ & 8 & NE & 8 & NE \\
\hline Spatial Supraspan Learning & NV $23.18 \pm 5.67$ & 20.33 & $9 \S$ & $15.34 \S$ & $9.41 \S$ \\
\hline Rey-Osterrieth Complex Figure, copy & $\leq 29$ & NE & $34 / 36$ & 35 & $34 / 36$ \\
\hline Rey-Osterrieth Complex Figure, recall & $\leq 15$ & NE & $11 \S$ & 19.5 & $12 \S$ \\
\hline copy of drawings & $<8 / 14$ & 13 & NE & 13 & NE \\
\hline limb praxis & NV $19.95 \pm 0.3$ & $18 \S$ & 19.75 & $17 \S$ & 19.75 \\
\hline orofacial praxis & NV $19.91 \pm 0.42$ & 19.75 & 19.75 & 19.75 & 19.75 \\
\hline body part denomination & $<8 / 12$ & $12 / 12$ & $12 / 12$ & $12 / 12$ & $12 / 12$ \\
\hline finger denomination & $<3 / 5$ & $5 / 5$ & $5 / 5$ & $5 / 5$ & $5 / 5$ \\
\hline \multicolumn{6}{|l|}{ neglect assessment } \\
\hline line bisection task (mm)ף & $>10$ & 1.6 & 6 & 2 & $16.6 \S$ \\
\hline Albert Cancellation Test & $>5$ & 0 & 0 & 0 & 0 \\
\hline Diller Cancellation Test & $>10$ & 0 & 0 & 0 & NE \\
\hline Bells Cancellation Test & $>5$ & 0 & 1 & 0 & 3 \\
\hline
\end{tabular}

* NE = not executed; NV = normative value (mean \pm standard deviation); STAI-Y = State-Trait Anxiety Inventory Form $Y$.

$\dagger$ A score above or below (as specified by the sign) the listed value was considered impaired.

$\ddagger$ A value below $40 / 100$ indicates left-handed dominance.

$\S$ Impaired performance.

If Line $20 \mathrm{~cm}$ long.

$\mathrm{mm})$, leaving tumor behind and a residual essential site (Label 3 in Fig. 2B). Tumor removal ended 34 minutes after resection of the essential site, obtaining a considerable opportunity to improve the tumor resection.

Postoperative Course. Postoperative T2-weighted MR images showed 70\% tumor removal. At the postoperative assessment, visuospatial memory tests remained unchanged, and the line bisection task revealed spatial neglect that disappeared 3 days later. The patient had no depression or anxiety at the preoperative and postoperative assessments. Histological examination revealed a WHO Grade II oligodendroglioma with deletions on both $1 \mathrm{p}$ and $19 \mathrm{q}$.

\section{Discussion}

The basic concept of neurophysiological cognitive mapping was described by Ojemann et al. ${ }^{8}$ who defined "essential" for language any area having significant func- tional derangement while stimulated during the naming task, that is, at least 2 of 3 times. These areas must be preserved, since the incidence and duration of postoperative impairment are significantly associated with the distance of the area from the site of tumor resection. ${ }^{5}$ Furthermore, the multiplicity of these areas in the same patient and the lack of interposing functional tissue for the same function led Ojemann and colleagues ${ }^{8}$ to describe them as mosaics. This model was confirmed and implemented by many authors who added other observations while using the same methods introduced by Ojemann et al. Later, the critical importance of the distance between functional areas and resection areas was also demonstrated for the motor circuit at both the cortical and the subcortical level.5,6

Although tempting, the idea that a functional area should be preserved with some buffer (millimeters) around it never proved to be a general assumption for intraoperative neurophysiological mapping. While this assumption is likely to be valid for primary sensory and 


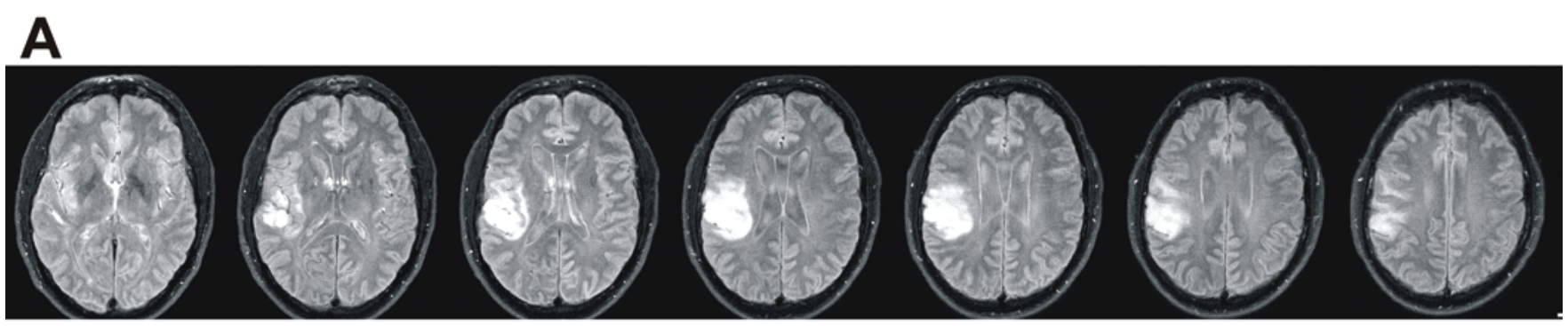

B

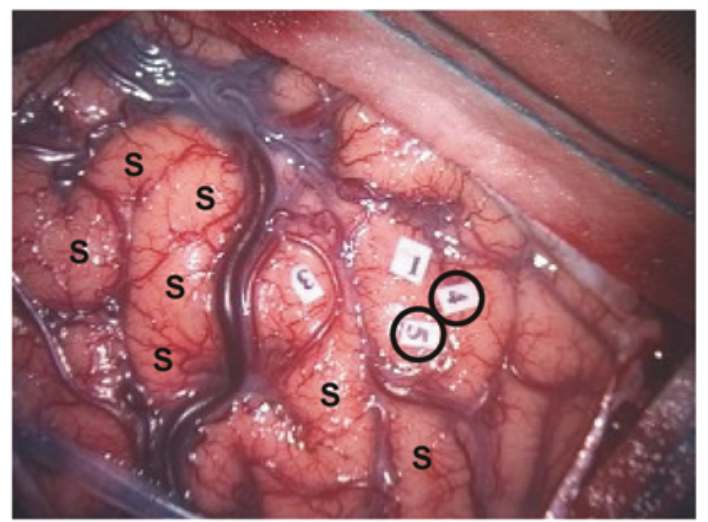

5

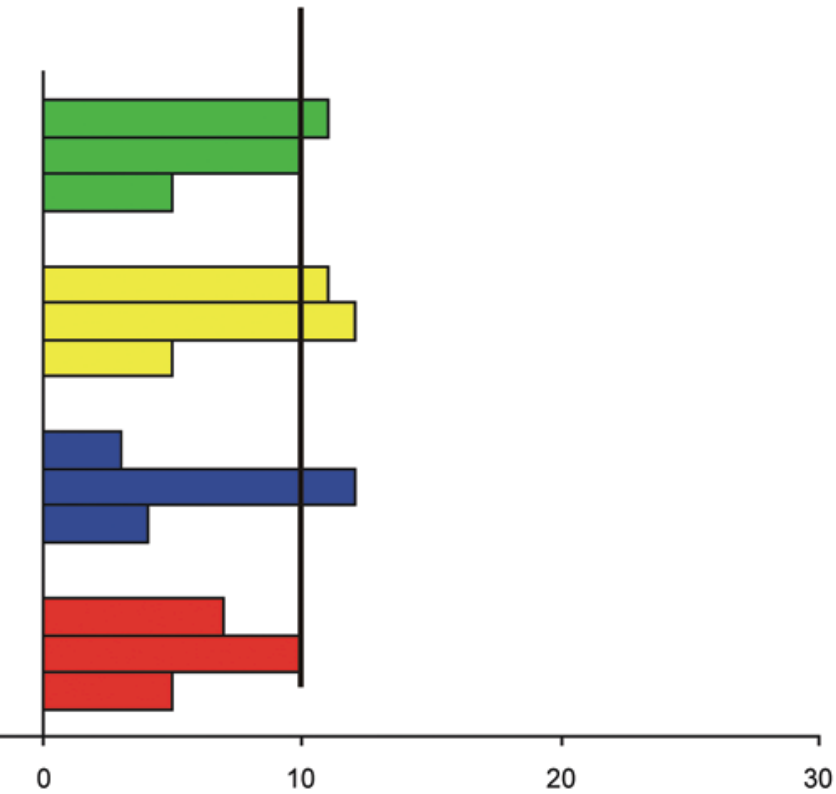

Deviation (mm)

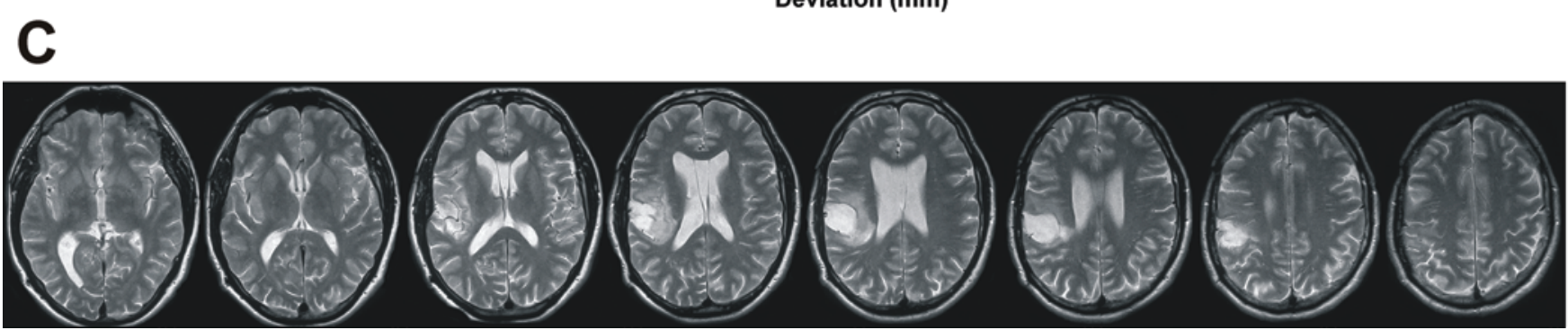

FIG. 1. Case 1. Preoperative FLAIR MR images (A) demonstrating a right parietal hyperintense area signifying extension of the infiltrating tumor. Photograph (B) showing sites for electrocortical stimulation (S). Only areas that produced at least 1 positive response on stimulation are indicated by numbered labels, which were positioned by the neurosurgeon prior to surgery. The circles around Labels 4 and 5 indicate essential areas, that is, areas at which at least 2 positive responses were obtained with rightward deviation of $10 \mathrm{~mm}$ or more. Bar graph depicting results of the line bisection task for each of the stimulated areas producing at least 1 positive response. Postoperative T2-weighted MR images (C) showing the resected area.

motor areas based on easily delineated cytoarchitectonics and functional grounds, it is disputable for secondary association areas. ${ }^{6}$ Within association areas, there is an additional and substantial difference between unimodal and heteromodal areas. ${ }^{7}$ The concept of discrete areas can be assumed only by exploring adjacent brain areas, concluding that different "nodes" or "epicenters" are strategic for a specific clinical task, which has a corresponding specific impairment. While the unimodal areas may respond to a simple task like picture naming, the heteromodal areas comprise a wide spectrum of integrated unimodal representations and the transformation of different perceptions into recognition. Evocative memory, hallucination, vocalization, induced emotional disorders, and many other evoked responses do not necessarily indicate a discrete essential area, neither do transient provoked focal clinical derangements (stimulation-induced error in task-related evoked responses) even after repetition of the same response in the same area.

To date, the concept of essential areas is critical for both the oncological and the functional approach, since its misunderstanding may limit surgery and/or lead to false-negative results. For language, there are those areas whose preservation guarantees good communication, that 


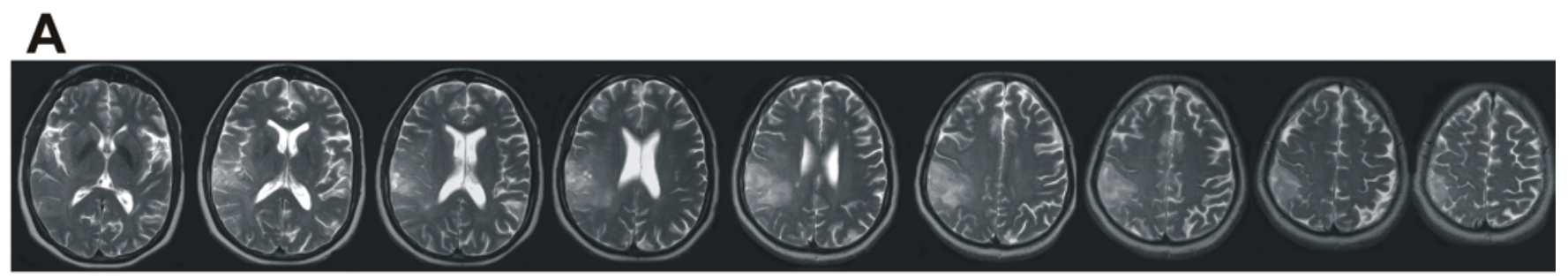

B

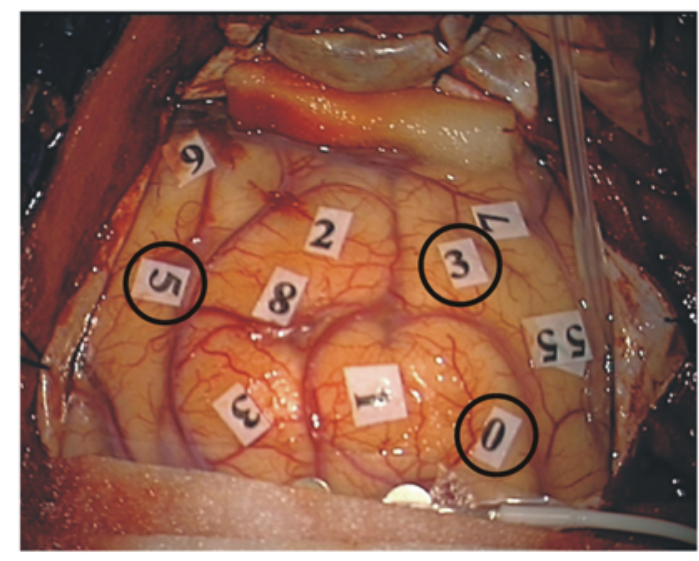

$-20$
$-30$

$-20$

$-10$

$-10$

\section{C}

Line Bisection Task

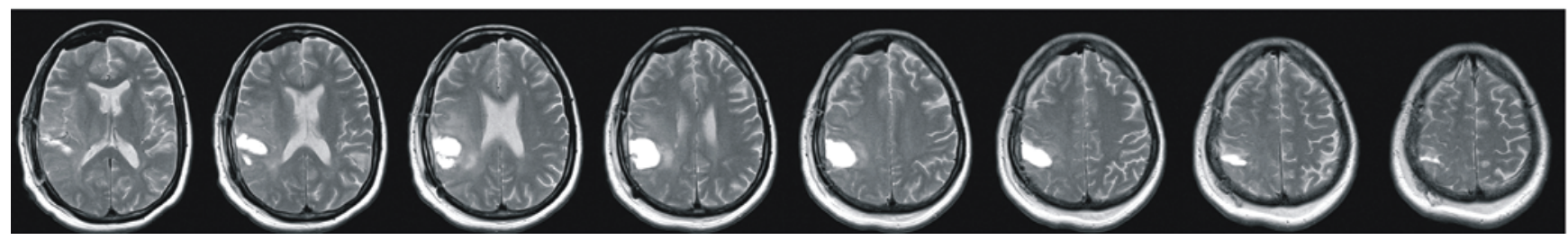

Fig. 2. Case 2. A: Preoperative T2-weighted MR images showing a right parietal hyperintense area with very irregular margins. B: Photograph showing sites for electrocortical stimulation. Here, all the labels positioned prior to stimulation are left in place. The circles around Labels 0,3 , and 5 indicate essential areas. Bar graph depicting results of the line bisection task for each of the stimulated areas producing at least 1 positive response. C: Postoperative T2-weighted MR images showing the resected area.

is, the maintenance of usable function, which is a broad category. In research, the ability to recognize more complex aspects such as language organization (grammar or syntax) or functions articulated through large-scale networks (memory, decision making, and spatial awareness) using a neurophysiological technique has not yet been demonstrated. Whether this is due to a lack of appropriate tasks or to an inherent limitation of the technique, which has a focal application, is not well established and is also beyond its clinical purpose.

In the featured cases, we observed that the method used for language mapping could not be applied to spatial awareness. An alternative could be to use a specific task, selected for its feasibility and robustness, to recognize the hierarchical organization of the function first under brain stimulation and then by clinically monitoring per- formance without stimulation, while having predefined criteria to stop the operation.

The definition and organization of nodes and modules are still controversial and under discussion. We have found that in this distributed heteromodal association network, the positive areas are not essential even under the traditional criteria used for language mapping; therefore, we suggest classifying them in order of importance (hierarchical organization). 2,16,17 This can have particular relevance for the surgeon when planning the operation, starting from safe areas and later moving on to more important areas to maximize tumor resection while maintaining online clinical feedback as well. Our experience suggested that this method is clinically meaningful (transient subclinical neglect persisted for only 3 days) and flexible (it allowed us to change the cutoff accordingly, possibly moving it for- 
ward to $10 \mathrm{~mm}$ ). A further advantage is the possibility to adapt monitoring to the complexity of brain surgery, which usually involves multiple critical areas in the same patient. This simple and rapid monitoring technique can control for more than one function, and we believe it could be extended at least to executive functions and memory.

One of the challenging issues is task selection. Bearing in mind that the aim is to prevent clinical impairment, we give priority to clinical impact rather than to exploring brain function in detail, which are different aims and almost opposing in nature. This implies striking a balance among sensitivity, specificity, and feasibility in one or more multipurpose tasks. To date, the line bisection task in spatial awareness seems to meet these requirements (specificity 90\%, sensitivity $60 \%$, and very good feasibility). ${ }^{13}$

\section{Conclusions}

It is likely that the classic criteria for language mapping to define an essential area cannot fit with large-scale networks. This has been proved by the observation that removing an essential site does not produce a permanent deficit. To prevent functional injury during tumor resection, new criteria must be devised using a neurophysiological setting. We propose a combined method with task-related evoked responses and nonevoked responses using one task.

\section{Disclosure}

The authors report no conflict of interest concerning the materials or methods used in this study or the findings specified in this paper.

Author contributions to the study and manuscript preparation include the following. Conception and design: Talacchi. Acquisition of data: all authors. Analysis and interpretation of data: all authors. Drafting the article: Talacchi. Critically revising the article: all authors. Reviewed submitted version of manuscript: all authors. Administrative/technical/material support: Santini. Study supervision: Talacchi, Savazzi.

\section{References}

1. Ben-Yishay Y, Diller L, Mandleberg I, Gordon W, Gerstman LJ: Differences in matching persistence behavior during block design performance between older normal and brain-damaged persons: a process analysis. Cortex 10:121-132, 1974

2. Driver J, Blankenburg F, Bestmann S, Ruff CC: New approaches to the study of human brain networks underlying spatial attention and related processes. Exp Brain Res 206:153-162, 2010

3. Duffau H, Capelle L, Sichez J, Faillot T, Abdennour L, Law Koune JD, et al: Intra-operative direct electrical stimulations of the central nervous system: the Salpêtrière experience with 60 patients. Acta Neurochir (Wien) 141:1157-1167, 1999

4. Emanuele B, Santini B, Talacchi A, Gerosa M, Savazzi S: Pre- and post-operative assessment of visuo-spatial functions in right hemisphere tumour patients: a pilot study. J Neurooncol 108:261-267, 2012

5. Haglund MM, Berger MS, Shamseldin M, Lettich E, Ojemann GA: Cortical localization of temporal lobe language sites in patients with gliomas. Neurosurgery 34:567-576, 1994

6. Keles GE, Lundin DA, Lamborn KR, Chang EF, Ojemann G, Berger MS: Intraoperative subcortical stimulation mapping for hemispherical perirolandic gliomas located within or adjacent to the descending motor pathways: evaluation of morbidity and assessment of functional outcome in 294 patients. J Neurosurg 100:369-375, 2004

7. Mesulam MM: Spatial attention and neglect: parietal, frontal and cingulate contributions to the mental representation and attentional targeting of salient extrapersonal events. Philos Trans R Soc Lond B Biol Sci 354:1325-1346, 1999

8. Ojemann GM, Ojemann JM, Lettich E, Berger M: Cortical language localization in left, dominant hemisphere. An electrical stimulation mapping investigation in 117 patients. J Neurosurg 71:316-326, 1989

9. Russell SM, Elliott R, Forshaw D, Kelly PJ, Golfinos JG: Resection of parietal lobe gliomas: incidence and evolution of neurological deficits in 28 consecutive patients correlated to the location and morphological characteristics of the tumor. $\mathbf{J}$ Neurosurg 103:1010-1017, 2005

10. Sack AT: Parietal cortex and spatial cognition. Behav Brain Res 202:153-161, 2009

11. Sanai N, Mirzadeh Z, Berger MS: Functional outcome after language mapping for glioma resection. N Engl J Med 358: $18-27,2008$

12. Santini B, Talacchi A, Casagrande F, Casartelli M, Savazzi S, Procaccio F, et al: Eligibility criteria and psychological profiles in patient candidates for awake craniotomy: a pilot study. J Neurosurg Anesthesiol 24:209-216, 2012

13. Schenkenberg T, Bradford DC, Ajax ET: Line bisection and unilateral visual neglect in patients with neurologic impairment. Neurology 30:509-517, 1980

14. Talacchi A, Santini B, Savazzi S, Gerosa M: Cognitive effects of tumour and surgical treatment in glioma patients. J Neurooncol 103:541-549, 2011

15. Talacchi A, Turazzi S, Locatelli F, Sala F, Beltramello A, Alessandrini F, et al: Surgical treatment of high-grade gliomas in motor areas. The impact of different supportive technologies: a 171-patient series. J Neurooncol 100:417-426, 2010

16. Tomasi D, Volkow ND: Association between functional connectivity hubs and brain networks. Cereb Cortex 21:20032013, 2011

17. Wig GS, Schlaggar BL, Petersen SE: Concepts and principles in the analysis of brain networks. Ann N Y Acad Sci 1224: 126-146, 2011

\section{Manuscript submitted October 23, 2012.}

Accepted December 17, 2012.

Please include this information when citing this paper: DOI: 10.3171/2012.12.FOCUS12358.

Address correspondence to: Andrea Talacchi, M.D., Institute of Neurosurgery, Department of Neurosciences, University Hospital, P. Stefani 1,37126 Verona, Italy. email: andrea.talacchi@univr.it. 